controlled endoscopically in $0.6 \%$ of patients who subsequently required surgery. Time of endoscopy was not associated with mortality $(\mathrm{p}=0.840)$, however inpatients had a higher 30 day mortality than outpatients $(8.7 \%$ vs $1.3 \%$, $\mathrm{p}<0.0005)$.

Trainees performed more endoscopies in 2018 than in 2011 (22.9\% vs $15 \%$ respectively) with no difference in mortality compared to consultants $(p=0.72)$. Trainees were on average in specialty training year (ST) 6 , but $41.4 \%$ of trainees were ST7. There was no association with year of training and mortality $(\mathrm{p}=0.146)$.

Conclusion There has been an increase in trainee experience of UGIB endoscopy since introduction of our training interventions, targeted at late years of training. The new training pathway will be four years as opposed to five and therefore it is likely to reduce the experience of trainees in managing UGIB which still has a significant mortality association. Our interventions have demonstrated that training can be improved with targeted approaches.

\section{P402 SMALL BOWEL ENDOSCOPY: DO WE OFFER ENOUGH TRAINING?}

Suneil A Raju*, Stefania Chetcuti Zammit, David S Sanders, Reena Sidhu. Academic Unit Of Gastroenterology, Sheffield, Uk, Sheffield, UK

\subsection{6/gutjnl-2020-bsgcampus.476}

Background There are currently 12 centres offering device assisted endoscopy (DAE) in the UK and between 30-35 offering video capsule endoscopy (VCE). There is a paucity of data on those offering training. We therefore quantify the training provided in small bowel endoscopy (SBE) across the UK to assess future training requirements.

Methods Online surveys and targeted calls to SBE centres were conducted of all British Society of Gastroenterology members in the UK to establish whether they were in SBE training and what level of training was offered to them.

Results From 17 centres there were 22 responses from gastroenterology fellows, trainees and consultants (36.4\%, 18.2\%, $45.5 \%$ respectively). Of all responders, $95.4 \%$ were independent in gastroscopies and $90.9 \%$ colonoscopies.

Training centres:

In total, $86.4 \%$ of centres offered VCE with 3 (IQR: 2-4) endoscopists per site interpreting videos. DAE was available in $72.7 \%$ of centres performed by 2 endoscopists (IQR: 2-3) per centre. Single and double balloon endoscopy was performed in $64.7 \%$ and $35.3 \%$ respectively under conscious sedation, deep sedation and both $(35.3 \%, 29.4 \%, 35.3 \%$ respectively).

Training in video capsule endoscopy:

VCE was interpreted by $63.6 \%$ of responders of which $78.6 \%$ were independent. $31.8 \%$ of responders were undergoing training in both VCE and DAE, 36.3\% in VCE and $9.1 \%$ in DAE. Of those who did not regularly review VCE, $75 \%$ were interested in becoming proficient.

Physicians required 50 (IQR: 20-50 videos) VCEs to gain competency. All physicians were confident in identifying pathology. To become independent, 50 videos (IQR 25-70) were reviewed. Responders who had attended VCE courses felt more confident in identifying pathology $(100 \%$ vs $33.3 \% \mathrm{p}=$ 0.002).

Training in device assisted endoscopy:

Only $36.4 \%$ of individuals undertook DAE of which $75 \%$ were independent. However $42.9 \%$ were interested in becoming proficient. On average, participants completed 55 (IQR: 19-85) procedures prior to being independent taking 12 months (IQR: 6-27 months). The target lesion was reached in $50-100 \%$ of cases. All DAE trainees performed therapeutic procedures. Moderate to severe pain was reported in $10 \%$ of patients under conscious sedation and no sedation related complications reported. The learning curve for antegrade DAE was easier than retrograde DAE. The terminal ileal intubation rate during retrograde DAE varied from less than 50\% to greater than $90 \%$.

Conclusion Training offered in SBE is heterogenous with individuals having different levels of prior experience. There is a need to offer and formalise VCE and DAE training to ensure uniform competence. However, centres must have set requirements to achieve prior to being able to offer training to ensure the training offered is up to standard.

\section{P403 OUTCOMES FROM THE SECOND IMPROVING SAFETY AND REDUCING ERROR IN ENDOSCOPY (ISREE) WORKSHOP}

${ }^{1,2,3}$ Srivathsan Ravindran*, ${ }^{2}$ Manmeet Matharoo, ${ }^{1}$ Tim Shaw, ${ }^{1}$ Eva Lynch,
${ }^{1}$ Raphael Broughton, $1,2,3$ Siwan Thomas-Gibson. ' ${ }^{1}$ oint Advisory Group on Gastrointestinal
Endoscopy (JAG), Royal College of Physicians, London; ${ }^{2}$ St Mark's Hospital; ${ }^{3}$ Imperial
College London

10.1136/gutjnl-2020-bsgcampus.477

Introduction The JAG 'Improving Safety and Reducing Error in Endoscopy' strategy was created to improve patient safety in endoscopy. A one day ISREE workshop was held to deliver

\begin{tabular}{|c|c|c|c|c|}
\hline Domain & Statement & $\begin{array}{l}\text { Pre- } \\
\text { Workshop } \\
\text { median }\end{array}$ & $\begin{array}{l}\text { Post- } \\
\text { Workshop } \\
\text { median }\end{array}$ & $p$ value \\
\hline 1 & I have an understanding of the ISREE strategy and how it reflects safety in my area of work & 5 & 9 & $<0.01$ \\
\hline 2 & I am aware of the 'case of the month' series and understand how it can be used to share learning & 8 & 10 & 0.03 \\
\hline 3 & I understand the elements of the safety domain of the GRS and how ISREE complements this & 6 & 8 & 0.02 \\
\hline 4 & I have an understanding of how endoscopy patient feedback is utilised for safety-related training & 5 & 8 & 0.01 \\
\hline 5 & I have an understanding of how incident reporting occurs across UK endoscopy services & 5 & 8 & 0.01 \\
\hline 6 & I am aware of current human factors endoscopy training strategies being developed in the UK & 4 & 9 & $<0.01$ \\
\hline 7 & I understand how JAG is utilising technology to support learning from incident & 5 & 8 & 0.01 \\
\hline 8 & I am confident in my knowledge of strategies to detect and support underperformance in endoscopy & 6 & 8 & $<0.01$ \\
\hline
\end{tabular}


training on safety-related issues in endoscopy, provide an update on ISREE activity and develop new ideas for future strategies.

Methods The workshop consisted of didactic and interactive sessions. Didactic sessions focused on safety metrics in endoscopy, simulation training, learning from incident and anaesthesia in endoscopy. Facilitated group sessions reviewed key areas where renewed focus was required: utilising patient feedback, digital innovation and supporting colleagues.

An interactive electronic voting system and field notes were used to collect data. Measured outcomes were pre and postworkshop knowledge of ISREE-related patient safety topics and self-rated confidence scores against 8 pre-defined statements. Wilcoxon signed rank test was used to assess differences in scores. Thematic analysis was conducted on transcribed data from group discussion and participant feedback.

Results There were 22 attendees from a multidisciplinary background, including gastroenterologists, surgeons, nurses, trainees and human factors/patient safety experts. Globally, there was significant improvement in knowledge scores $(p<0.001)$ and confidence scores following the workshop (see table 1 ).

Three major themes were identified: developing repositories of good practice through patient feedback, standardising digital referral data and developing coaching and mentoring strategies to support colleagues. The majority (90.9\%) of participants felt that the workshop had increased their understanding of strategies to improve safety in endoscopy.

Conclusions The ISREE workshop improved knowledge and confidence in areas of patient safety relevant to endoscopy, with positive engagement from participants. Novel ideas were generated that have informed current and future ISREE strategies.

\section{P404 JAG CORE ENDOSCOPY PROGRAMME: A DESCRIPTIVE STUDY OF E-LEARNING ENGAGEMENT}

\footnotetext{
1,2,3 Srivathsan Ravindran* ${ }^{2,3}$ Siwan Thomas-Gibson, 'Raphael Broughton, 'Mark Coleman, ${ }_{1,4}^{1, G e o f f ~ S m i t h . ~}{ }^{1}$ Joint Advisory Group on Gastrointestinal Endoscopy (JAG), Royal College of Physicians, London; ${ }^{2}$ St Mark's Hospital; ${ }^{3}$ Imperial College London; ${ }^{4}$ Health Education UK
}

\subsection{6/gutjnl-2020-bsgcampus. 478}

Aim The Joint Advisory Group on Gastrointestinal Endoscopy (JAG) core endoscopy e-learning course is designed to provide essential basic training to all endoscopy staff. This study is the first to analyse learners' use of the e-learning platform and engagement with content.

Method Data was extracted from the e-learning for healthcare (e-LFH) platform between Dec 2015 and Dec 2019. The core endoscopy course has 11 individual modules. Data regarding user type, user activity and engagement were collated. Outcomes variables were time per session and completion rates. Descriptive statistics are reported as median and interquartile range (IQR). Statistical differences were assessed using the Kruskal-Wallis test and correlation by Spearman's rank.

Results Between December 2015 and 2019, there were 1835 users of the core endoscopy programme. The top 3 regions based on users were: Midlands (20.6\%), North East and Yorkshire (14.9\%) and London (12.4\%). There was a median of 32 new users per month (IQR 19.5 - 47.5) and 47 active users per month (IQR 30.5 - 71.0). Users included nurses (47.5\%), training doctors (16.1\%), non-training doctors (11.2\%) and healthcare assistants (HCAs; 2.9\%). There was no significant difference in active users by month $(p=0.88)$.

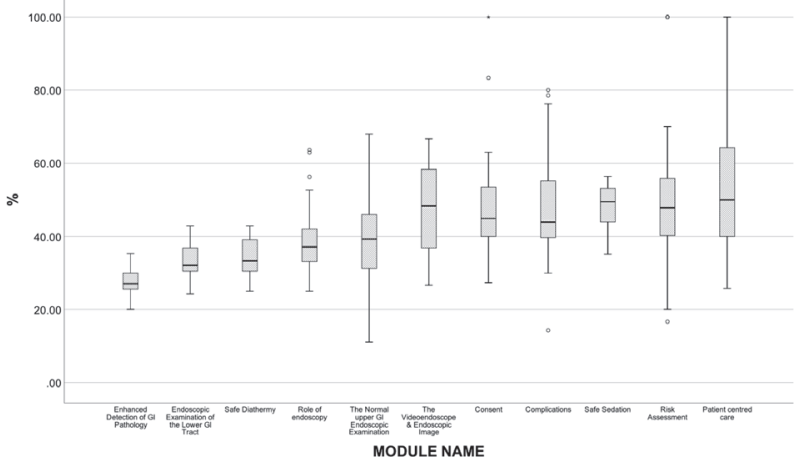

Abstract P404 Figure 1

There were 12,122 session launches with a median of 28 session launches per month (IQR 17.0 - 46.0). The 'role of endoscopy' was the most launched module with a median of 54 launches per month (IQR 31 - 81). Globally, the median completion rate was $42.1 \%$ per module (IQR 35.2 - 50.0), which is lower than other similar e-LFH modules (median 48-52\%). There were significant differences in completion between modules $(p<0.001)$ with 'patient centred care' having the highest median completion rate $(50.0 \%)$, as shown in figure 1:

The median time spent per module was 21 minutes (IQR $16-28)$ but there was significant variability in the amount of time spent per module $(p=0.03)$. There was no significant correlation between module completion rate and time spent $(p$ $=0.05)$ or number of assessment questions $(p=0.12)$, but weak correlation with module length $\left(r_{s} 0.35, p<0.001\right)$.

There were significant differences between user types and duration of activity $(p=0.001)$, with HCAs spending the least amount of time per session (median 10 mins, IQR 1 20). However, there was no significant difference between staff type and completion rate $(p=0.56)$.

Conclusions There are comparably low completion rates and variability in activity by user groups. These results will inform further changes to the e-LFH endoscopy programme particularly in promoting module completion and improving engagement with specific modules.

\section{P405 ENDOSCOPY IN-SITU SIMULATION: EVALUATION OF A NOVEL PROGRAMME IN A TERTIARY ENDOSCOPY UNIT}

${ }^{1,2,3}$ Srivathsan Ravindran* ${ }^{1}$ Adam Haycock, ${ }^{1}$ Adam Humphries, ${ }^{3}$ Hutan Ashrafian,

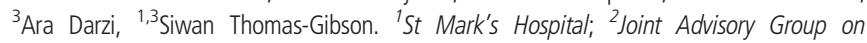
Gastrointestinal Endoscopy (JAG), Royal College of Physicians, London; ${ }^{3}$ Imperial College London

\subsection{6/gutjnl-2020-bsgcampus.479}

Introduction We developed a pilot programme to learn from adverse events in our unit, using in-situ simulation (ISS). Our programme focuses on Endoscopic Non-Technical Skills (ENTS) development as a means to improve learning.

Methods A pilot study was designed to evaluate outcomes from the first year of our ISS programme. We conducted a pilot high-fidelity, multidisciplinary ISS session followed by debrief, based on an established simulation model. Faculty included a simulated patient, consultant endoscopist, resuscitation officer and research fellow with a simulation interest. 